\title{
Localization of Plastic Deformation and Fracture in Aluminum Polycrystals
}

\author{
N. V. Zarikovskaya, ${ }^{1, a}$ and L. B. Zuev ${ }^{2, b}$ \\ ${ }^{1}$ Institute of Strength Physics and Materials Science, Siberian Branch of the Russian Academy of \\ Sciences, Tomsk, Russia \\ ${ }^{2}$ Tomsk State University of Control Systems and Radioelectronics, Tomsk, Russia \\ a chepko-znv@mail.ru, b1bz@ispms.tsc.ru
}

The effect of the grain size as a basic structural parameter on plastic strain macrolocalization has been studied for polycrystalline aluminum. The mathematical form of the above dependence has been verified. The limiting cases have been defined both for small-and coarse-grain ranges. The effect of sample dimension on the macrolocalization period has been considered.

Keywords: plastic deformation localization, polycrystalline aluminum, deformation curve, autowave, spatial period, failure.

Introduction. The plastic deformation of polycrystalline materials is an essential and often a defining factor in many technological processes. At present significant progress has been made in the physical theory of plasticity. A significant volume of experimental data on the distinctive features of deformation and fracture has been obtained for polycrystalline aluminum.

Plastic flow tends to localize at all the stages. The form of localization patterns varies from the yield limit to fracture depending on the prevailing law of work hardening.

Our experimental investigations suggest that the observed regularities exhibited by plastic flow are the result of self-organization of the deforming medium. According to Zuev and Danilov [1], the above regularities can be considered as waves of localized plastic deformation.

Experimental Procedure. The uniaxial tension tests were performed for a wide range of mono- and polycrystals using an Instron-1185 testing machine with load $F=$ $10 \mathrm{kN}$ and loading rate $\dot{\varepsilon}=3.3 \cdot 10^{-6} \mathrm{~m} / \mathrm{s}$.

Localized strain zones on the test specimen were revealed by the method of double-exposure speckle interferometry [1], which yields distributions of plastic strain tensor components [1].

Out of five types of deformation localization patterns only three are observed on the flow curve of polycrystalline aluminum, namely:

- At the stage of linear work hardening a set of mobile nuclei of localized plastic deformation originates in the test specimen and moves in a regular fashion, thereby forming a running wave.

- At the stage of parabolic work hardening a set of immobile nuclei of deformation localization emerges in the test specimen.

- At the pre-failure stage localized plastic deformation nuclei merge together, resulting in necking and viscous failure of the test specimen.

The distribution patterns of plastic strain tensor components are shown $\varepsilon_{x x}$ in Fig. 1 for polycrystalline aluminum both at the linear and the parabolic work hardening stage.

The observed regularities of plastic flow localization are common to all deforming materials $[1,2]$. It is found that plastic deformation tends to localize in certain zones of the deforming specimen and is characterized by macroscopic scale, i.e., wavelength $\lambda$.

It has been found that $\lambda$ depends on material parameters, i.e., length scale, crystal lattice geometry, grain size, etc. Therefore, to determine the dependence of $\lambda$ on the grain size and dimensions of a polycrystalline material is of particular interest. 

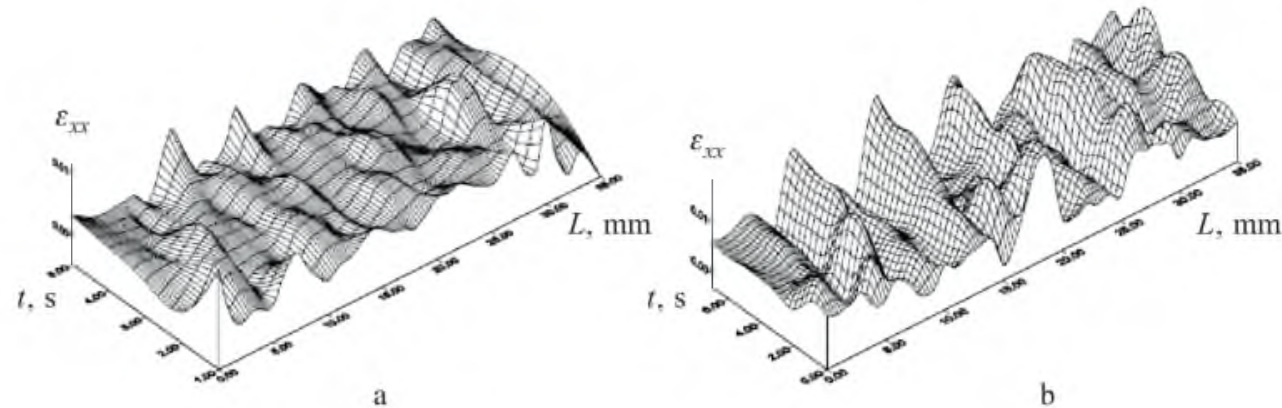

Fig. 1. Space-time distributions of the elongation component obtained for polycrystalline aluminum having a grain size $D=190 \mu \mathrm{m}$ : (a) linear stage at $\varepsilon=4.8-5.6 \%$; (b) parabolic stage at $\varepsilon=8.0-8.8 \%$.

The tests were conducted using A85 aluminum samples whose grain size was easily varied from 0.008 to $10 \mathrm{~mm}$ by the method of collective recrystallization.

Grain Size Dependence of Localization Wavelength for Polycrystalline Aluminum. Figure 2 shows $\lambda$ as a function of the grain size $D$. Numerical processing of the above dependence yields the following equation:

$$
d \lambda / d D=a \lambda-b \lambda^{2}
$$

where $a$ and $b$ are the positive dimensional constants [3].

The solution to the above equation is as follows:

$$
\lambda=\frac{\lambda_{0}}{1+C \exp (-a D)},
$$

where $\lambda_{0}=a / b$, and $C$ is a non-dimensional integration constant.

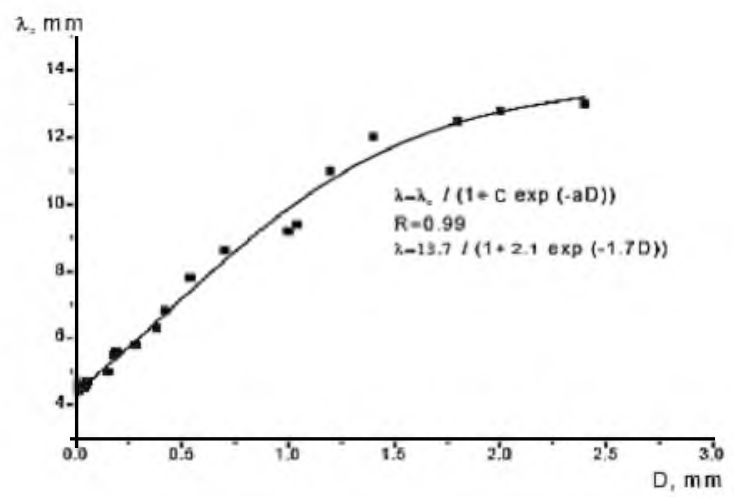

Fig. 2. Wavelength $\lambda$ dependence on the grain size $D$ for polycrystalline aluminum.

Equation (2) describes, with a sufficient accuracy, a set of experimental $\lambda(D)$ data in a wide interval of $D$ values (correlation coefficient $R=0.98$ ). The curve in Fig. 2 may be subdivided into three portions [3]: 1) as $D$ goes up to $0.5 \mathrm{~mm}, \lambda$ grows exponentially up to $\lambda e^{a D}$ (Fig. 3a); 2) in the range $0.5 \leq D \leq 2.5 \mathrm{~mm}$ the dependence takes on the logarithmic form $(\lambda \ln D)$ (Fig. 3b); 3) at $D \geq 2.5 \mathrm{~mm}, \lambda$ becomes constant $\left(\lambda \rightarrow \lambda_{0} \approx\right.$ $15 \mathrm{~mm})$. 

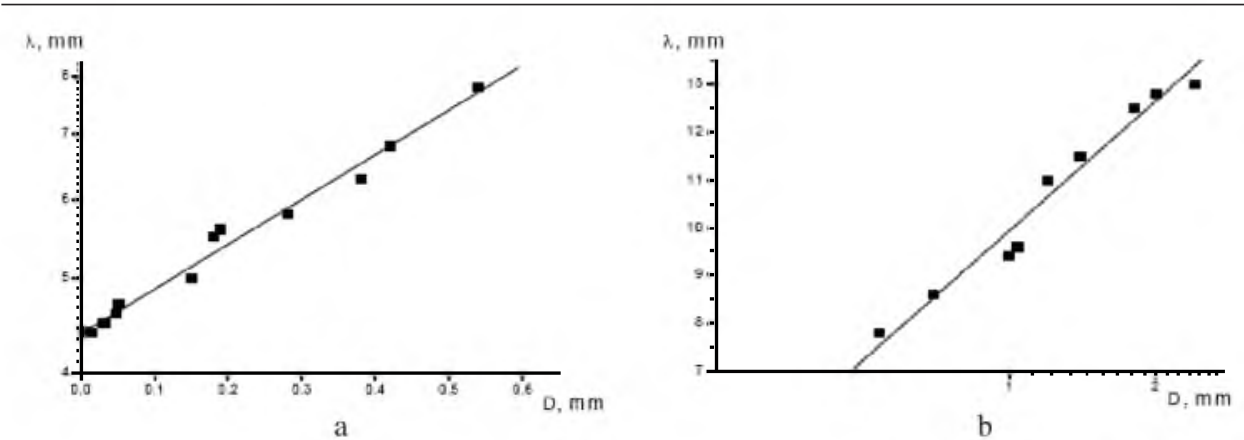

Fig. 3. Limiting cases of the wavelength dependence on grain size: (a) $D \leq 0.5 \mathrm{~mm}$; (b) $D \geq 0.5 \mathrm{~mm}$.

The effect of sample geometry (in particular, the sample thickness) on the macrolocalization period was examined for aluminum samples having grain size $D \leq 0.5 \mathrm{~mm}$. It can be seen in Fig. 4 , with increasing sample thickness, $\lambda$ grows as well

Numerical processing of experimental data yielded constants $a=1.1 \mathrm{~mm}^{-1}$ and $b=0.2 \mathrm{~m}^{-2}$ for $2 \times 10 \times 50-\mathrm{mm}$ samples and $a=1.5 \mathrm{~mm}^{-1}$ and $b=0.2 \mathrm{~m}^{-2}$ for $5 \times 10 \times 50-\mathrm{mm}$ samples. Evidently, $b$ is unaffected by the sample thickness.

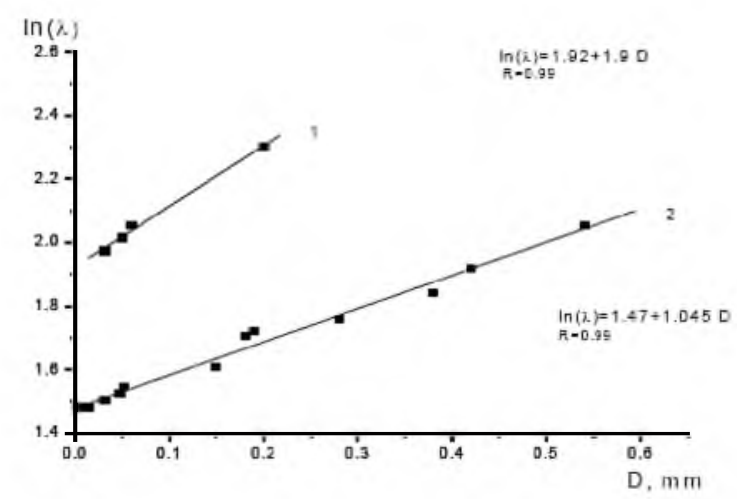

Fig. 4. Grain size dependence of macrolocalization periods on the sample thickness: lines 1 and 2 correspond to $t_{1}=5 \mathrm{~mm}$ and $t_{2}=2 \mathrm{~mm}$, respectively.

Distinctive Features of Deformation Macrolocalization at the Prefracture Stage. In order to get a holistic picture of deformation for polycrystalline aluminum, the final stage of the process, i.e., the prefracture stage, has been explored. It was shown earlier [4] that the most striking feature of the plastic deformation localization reveals itself at the latter stage.

The prefracture stage is a parabolic one, i.e., the stress-strain dependence for this stage has the form $\sigma \sim \varepsilon^{n}$ (where $n$ is the parabola exponent). It has been shown that with $n<0.5$ the localized deformation nuclei move along the sample at a velocity $V$ [4],

$$
V(n)=V_{0}(n-q)^{2} .
$$

At the parabolic stage $(n \approx 0.5)$ the localized deformation nuclei become motionless $(V=0)$, while at the linear stage $(n=1)$ they move synchronously with different velocities.

The nuclei locations were plotted in the $X(t)$ or $X(\varepsilon)$ coordinates, where $X$ is the nucleus' coordinate, $t$ is the deformation time, and $\varepsilon$ is the deformation (Fig. 5). 


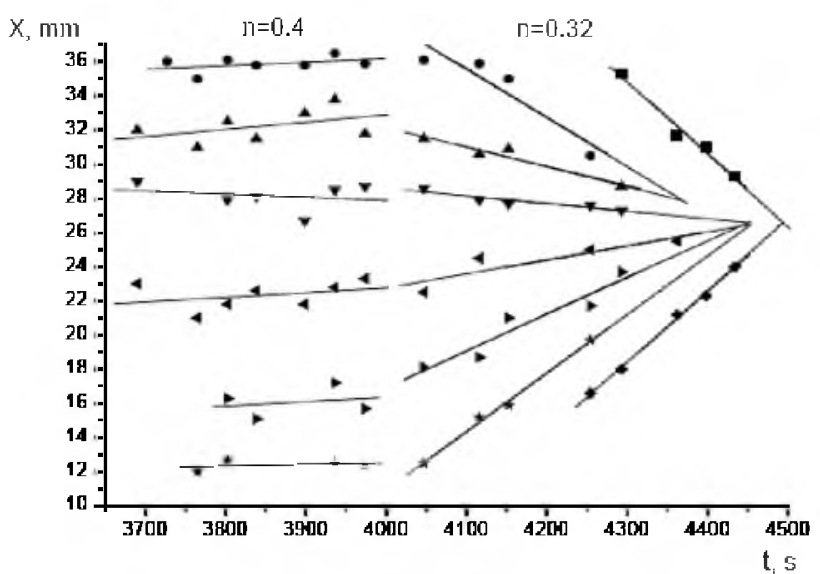

Fig. 5. Positions of localization nuclei vs. time.

It can be seen from the plot that with $n \leq 0.4$ the nuclei's trajectories would form a bundle whose pole pinpoints the location of future fracture.

The velocity of a nucleus can be defined from the slope of the straight line. Also, it should be noted that the nuclei move with different velocities, some of them disappearing altogether.

Thus, one can predict the place of future fracture long before the beginning of visible necking.

1. L. B. Zuev and V. I. Danilov, Phil. Mag. A, 79, 43 (1999).

2. L. B. Zuev, Ann. Phys., 3, 965 (2001)

3. L. B. Zuev, B. S. Semukhin, and N. V. Zarikovskaya, Int. J. Solids Struct., 40, 941 (2003).

4. L. B. Zuev and V. I. Danilov, Tech. Phys., 50, 1636 (2005). 